Pacific Journal of Mathematics

INSTATIONARY NAVIER-STOKES EQUATIONS AND 


\title{
INSTATIONARY NAVIER-STOKES EQUATIONS AND PARABOLIC SYSTEMS
}

\author{
WOLF VON WAHL
}

This paper mainly deals with instationary Navier-Stokes equations in two space dimensions. We derive the regularity results which are known to be valid for the corresponding linear system. Moreover, our method applies to parabolic semilinear systems with one-sided conditions.

o. Introduction and notation. This paper mainly deals with the strong solvability of the instationary Navier-Stokes equations

$$
\begin{aligned}
\boldsymbol{u}^{\prime}-\nu \Delta \boldsymbol{u}+\boldsymbol{u} \nabla \boldsymbol{u}+\nabla p & =\boldsymbol{f}, \\
\nabla \cdot \boldsymbol{u} & =0, \\
\boldsymbol{u} \mid \partial \Omega & =0, \\
\boldsymbol{u}(0) & =\boldsymbol{\phi}
\end{aligned}
$$

over a cylindrical domain $(0, T) \times \Omega . T$ is an arbitrary positive number, $\Omega$ is a bounded domain of $\boldsymbol{R}^{2}$ with smooth boundary. It is well known, (I) has a uniquely determined weak solution if the external force $\boldsymbol{f}$ is from $L^{2}((0, T) \times \Omega)$ and $\phi \in \dot{H}^{1}(\Omega)$ (these conditions can be weakened). But if one wants to show that the weak solution is a strong one, additional conditions are needed, concerning mainly the differentiability of $f$ with respect to $t$ or the regularity of $u$. Lions [5], Ch. 1 requires differentiability of $f$, as does Ladyzenskaja [3], Ch. 6. In one of their papers, Shinbrot and Kaniel [6], p. 313, require additional regularity of $\boldsymbol{u}$; Serrin [8] requires even more and then only obtains interior regularity of $u$. The method of Shinbrot and Kaniel [7] yields a strong solution under exactly the conditions we want, namely, $\boldsymbol{f} \in L^{2}\left((0, T), L^{2}(\Omega)\right)$ and $\boldsymbol{\phi} \in$ $\dot{H}^{1}(\Omega)$, but this solution is only local in time and may not exist, in general, in the full interval $0 \leqq t \leqq T$, unless $f$ and $\phi$ are small enough. Fujita and Kato [2] also obtain a strong solution, under even weaker conditions on $\boldsymbol{f}$ and $\phi$, but, again, their solution is only local. In this paper we show that none of these additional conditions is necessary and that for every $f \in L^{2}((0, T) \times \Omega), \phi \in \stackrel{\circ}{H}^{1}(\Omega)$ there exists a pair

$$
(\boldsymbol{u}, p) \in L^{2}\left((0, T), H^{2}(\Omega)\right) \cap L^{\infty}\left((0, T), \stackrel{\circ}{H}^{1}(\Omega)\right) \times L^{2}\left((0, T), H^{1}(\Omega)\right)
$$

with

$$
\boldsymbol{u}(0)=\boldsymbol{\phi},
$$




$$
\begin{gathered}
\boldsymbol{u}^{\prime} \in L^{2}\left((0, T), L^{2}(\Omega)\right), \\
\nabla \cdot \boldsymbol{u}=0,
\end{gathered}
$$

and

$$
\boldsymbol{u}^{\prime}-\boldsymbol{\nu} \Delta \boldsymbol{u}+\boldsymbol{u} \nabla \boldsymbol{u}+\nabla p=\boldsymbol{f}
$$

in the distributional sense. $\boldsymbol{u}$ is determined uniquely. If $\boldsymbol{f} \in L^{p}((0$, $T) \times \Omega), \boldsymbol{\phi} \in H^{2, p}(\Omega) \cap \dot{H}^{1, p}(\Omega),(\boldsymbol{u}, p)$ correspondingly is in $L^{p}((0, T)$, $\left.H^{2, p}(\Omega)\right) \times L^{p}\left((0, T), H^{1, p}(\Omega)\right), p>2$. The latter is not proved here since the proof follows the lines of the proof in the case $p=2$.

In the second chapter we prove an analogous result for parabolic systems

$$
\boldsymbol{u}^{\prime}+A(t) \boldsymbol{u}+f\left(t, x, \boldsymbol{u}, \nabla \boldsymbol{u}, \cdots, \nabla^{m} \boldsymbol{u}\right)=g,
$$

$A(t), 0 \leqq t \leqq T$, being elliptic operators of divergence structure of order $2 m$.

Of course instead of boundary values 0 one can treat the case where boundary values $\boldsymbol{a}$ are prescribed having the regularity properties of the solution expected.

We introduce some notation. Let $X$ be a Banach-space. $C^{\nu}([0, T], X)$ is then the Banach-space of all $\nu$-times continuously differentiable mappings from $[0, T]$ in $X . L^{p}((0, T), X), 1 \leqq p \leqq \infty$, is the Banach-space of all measurable mappings $u:(0, T) \rightarrow X$ with norm

$$
\left(\int_{0}^{T}\|u(t)\|_{X}^{p} d t\right)^{1 / p}, \quad \underset{0 \leqq t \leqq T}{\operatorname{ess} \sup _{0}}\|u(t)\|_{X} \text { respectively . }
$$

$\Omega$ is a bounded open set of $\boldsymbol{R}^{n} \cdot H^{\nu, p}(\Omega)$ is the Banach-space of all real valued functions defined on $\Omega$ with distributional derivatives in $L^{p}(\Omega)$ up to the order $\nu$. Its norm is denoted by

$$
\|\cdot\|_{\nu, p} \text { or }\|\cdot\|_{H^{\nu, p}(\Omega)} \text {. }
$$

As usual $\dot{H}^{\nu, p}(\Omega)$ is the completion of $C_{0}^{\infty}(\Omega)$ in the norm of $H^{\nu, p}(\Omega)$.

The norm of $C^{0}\left([0, T], H^{\nu, p}(\Omega)\right)$ is denoted by \|\|$_{\nu} \mid \|_{\nu, p}$. Frequently we will consider functions being continuous on $[0, T] \times \bar{\Omega}=: Q_{T}$. For those we introduce the notations

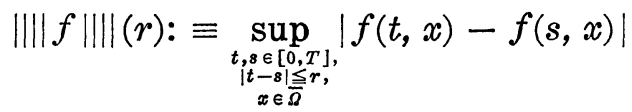

$$
\begin{aligned}
& +\sup _{\substack{t \in[0,0], x, y \in \bar{\Omega},|x-y| \leqq r}}|f(t, x)-f(t, y)|,
\end{aligned}
$$




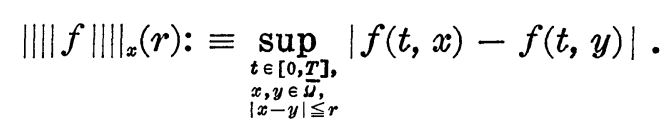

For $\sup _{(t, x) \in Q_{T}}|f(t, x)|$ we simply write $\||f|\| \cdot C^{\nu}(\bar{\Omega}), C^{\nu+\alpha}(\bar{\Omega})$ are the Banach-spaces of all complex valued functions defined on $\bar{\Omega}$, being continuously differentiable up to the order $\nu$ in $\Omega$ and having derivatives of order $\nu$ being continuous in $\bar{\Omega}$ or Hölder-continuous with exponent $\alpha$ in $\bar{\Omega}$. The norms of $C^{\nu}(\bar{\Omega}), C^{\nu+\alpha}(\bar{\Omega})$ are denoted by $\|\cdot\|_{\nu}$, $\|\cdot\|_{\nu+\alpha}$ respectively. Moreover we use the notation

$$
\begin{gathered}
\|\cdot\|_{p}:=\|\cdot\|_{L^{p(\Omega)}}:=\|\cdot\|_{o, p}, H^{\nu}(\Omega):=H^{\nu, 2}(\Omega), \stackrel{\circ}{H}^{\nu}(\Omega):=\dot{H}^{\nu, 2}(\Omega), \\
D_{j}:=\frac{1}{i} \frac{\partial}{\partial x_{j}}, \\
D^{\tilde{\alpha}}:=\prod_{\jmath=1}^{n} D_{j}^{\alpha j},
\end{gathered}
$$

where $\tilde{\alpha}$ is a multiindex with components $\alpha_{j} \in N \cup\{0\} . \quad N$ is the set of positive integers.

$\Omega$ or $\partial \Omega$ is said to be of class $C^{\nu}$ or $C^{\nu+\alpha}$ if $\partial \Omega$ locally admits a representation

$$
x_{k}=\phi\left(x_{1} \cdots, x_{k-1}, x_{k+1}, \cdots, x_{n}\right)
$$

with $\phi \in C^{\nu}$ or $C^{\nu+\alpha}$ respectively and if $\Omega$ locally is one one side of $\partial \Omega$. If no misunderstanding is possible we often write $X$ instead of $X^{\imath}, X$ a vector space. If $X$ has a scalar product (.,.) the scalar product of $X^{l}$, namely $\sum_{\lambda=1}^{l}(.,$.$) is denoted by (.,$.$) too.$

$D^{\prime}(\Omega)$ is the space of all distributions over $\Omega$. For functions $u \in\left(H^{1}(\Omega)\right)^{n}$ the element $\boldsymbol{u} \cdot \nabla \boldsymbol{u} \in \boldsymbol{R}^{n}$ is defined as the vector having the components

$$
\sum_{i=1}^{n} u^{i} \frac{\partial u^{\lambda}}{\partial x_{i}}, \quad 1 \leqq \lambda \leqq n
$$

( $\boldsymbol{u}$ has the components $u^{i}$ ). If $\boldsymbol{u} \in\left(H^{K}(\Omega)\right)^{l}$ and $A$ is a differential operator of order $K, A u$ means the vector $\left(A u^{1}, \cdots, A u^{l}\right)$.

1. Instationary Navier-Stokes equations. First we define the meaning of strong and weak solutions to the instationary NavierStokes system. For that purpose we assume that $\Omega$ is a bounded open set of $R^{n}, n=2$, having a boundary of class $C^{3}$. Let $\nu, T$ be arbitrary $>0$.

Definition. An element $(\boldsymbol{u}, p)$ of $L^{\infty}\left((0, T), L^{2}(\Omega)\right) \cap L^{2}((0, T)$, 
$\left.\stackrel{\circ}{H}^{1}(\Omega)\right) \times D^{\prime}\left(Q_{T}\right)$ with $\nabla \cdot \boldsymbol{u}(t)=0$ for almost all $t \in(0, T)$ is a weak solution to the problem

(NS)

$$
\begin{aligned}
\boldsymbol{u}^{\prime}-\boldsymbol{\nu} \Delta \boldsymbol{u}+\boldsymbol{u} \cdot \nabla \boldsymbol{u}+\nabla p & =\boldsymbol{f}, \\
\nabla \cdot \boldsymbol{u} & =0, \\
\boldsymbol{u} \mid \partial \Omega & =0, \\
\boldsymbol{u}(0) & =\boldsymbol{\phi},
\end{aligned}
$$

if

$$
\begin{aligned}
& -\int_{0}^{T}\left(\boldsymbol{u}(t), \boldsymbol{\psi}^{\prime}(t)\right) d t+\nu \int_{0}^{T}(\nabla \boldsymbol{u}(t), \nabla \boldsymbol{\psi}(t)) d t \\
& +\int_{0}^{T}(\boldsymbol{u}(t) \cdot \nabla \boldsymbol{u}(t), \boldsymbol{\psi}(t)) d t=\int_{0}^{T}(\boldsymbol{f}(t), \boldsymbol{\psi}(t)) d t+(\boldsymbol{\phi}, \boldsymbol{\psi}(0))
\end{aligned}
$$

for all test functions $\psi$ with

$$
\begin{aligned}
& \psi^{\prime} \in L^{2}\left((0, T), L^{2}(\Omega)\right), \\
& \psi \in L^{2}\left((0, T), \dot{H}^{1}(\Omega)\right), \\
& \nabla \cdot \psi=0, \psi(T)=0 .
\end{aligned}
$$

$f, \phi$ are fixed elements from $L^{2}\left((0, T) L^{2}(\Omega)\right)$ and $\dot{H}^{1}(\Omega)$ respectively. Moreover, $\nabla \cdot \boldsymbol{\phi}=0$. An element $(\boldsymbol{u}, p)$ of $V \times L^{2}\left((0, T), H^{1}(\Omega)\right)$ is a strong solution to the problem (NS) if the equations $\boldsymbol{u}^{\prime}-\boldsymbol{\nu} \Delta \boldsymbol{u}+$ $\boldsymbol{u} \nabla \boldsymbol{u}+p=\boldsymbol{f}, \nabla \cdot \boldsymbol{u}=0$ are fulfilled in the distributional sense and if $\boldsymbol{u}(0)=\boldsymbol{\phi}$. Here $V$ denotes the Banach space of all $u \in L^{2}((0, T)$, $\left.H^{2}(\Omega)\right) \cap L^{\infty}\left((0, T), \stackrel{\circ}{H}^{1}(\Omega)\right)$ with $u^{\prime} \in L^{2}\left((0, T), L^{2}(\Omega)\right)$ and norm

$$
\underset{0 \leq t \leqq T}{\operatorname{ess~sup}}\|u(t)\|_{1,2}+\left(\int_{0}^{T}\left\|u^{\prime}(t)\right\|_{L^{2}(\Omega)}^{2} d t+\int_{0}^{T}\|u(t)\|_{2,2}^{2} d t\right)^{1 / 2} .
$$

Observe that $\boldsymbol{u}(0)=\boldsymbol{\phi}$ makes sense since $\boldsymbol{u} \in V$ implies that $\boldsymbol{u} \epsilon$ $C^{\circ}\left([0, T], L^{2}(\Omega)\right)$. For any function from $C_{0}\left(\boldsymbol{R}^{2}\right)$ we have

$$
\begin{aligned}
u^{2}(x, y) & =2 \int_{-\infty}^{x} u(\xi, y) u_{x}(\xi, y) d \xi \\
& \leqq 2 \int_{-\infty}^{\infty}\left|u(\xi, y) \| u_{x}(\xi, y)\right| d \xi
\end{aligned}
$$

and also

$$
u^{2}(x, y) \leqq 2 \int_{-\infty}^{\infty}|u(x, \eta)|\left|u_{x}(x, \eta)\right| d \eta
$$

Multiplying these last two inequalities and integrating over $x$ and $y$, we find, by the Schwarz inequality,

$$
\|u\|_{L^{4}(\Omega)} \leqq 4 \sqrt{2}\|\nabla u\|_{L^{2}(\Omega)}^{1 / 2}\|u\|_{L^{2}(\Omega)}^{1 / 2}
$$


for all $u \in \dot{H}^{1}(\Omega)$. Then, by (I.1), it follows that

$$
\begin{aligned}
& |(\boldsymbol{u}(t) \cdot \nabla \boldsymbol{u}(t), \boldsymbol{\psi}(t))| \leqq\|\boldsymbol{u}(t)\|_{L^{4}(\Omega)}\|\nabla \boldsymbol{u}(t)\|_{L^{2}(\Omega)}\|\boldsymbol{\psi}(t)\|_{L^{4}(\Omega)}, \\
& \quad \leqq 2\|\nabla \boldsymbol{u}(t)\|_{L^{2}(\Omega)}^{3 / 2}\|\nabla \boldsymbol{\psi}(t)\|_{L^{2}(\Omega)}^{1 / 2}\|\boldsymbol{u}(t)\|_{L^{2}(\Omega)}^{1 / 2}\|\boldsymbol{\psi}(t)\|_{L^{2}(\Omega)}^{12}
\end{aligned}
$$

Thus all expressions occuring in the definition of the weak solution to the problem (NS) are well-defined.

As it is shown in [5], Chap. I, the problem (NS) always has a weak solution. Moreover, $\boldsymbol{u}$ is determined uniquely.

Our nonlinearity $\boldsymbol{g}(x, \boldsymbol{u}, \nabla \boldsymbol{u})=\boldsymbol{u} \cdot \nabla \boldsymbol{u}$ has the following properties: (NS 1): $\boldsymbol{g}$ is continuous from $\bar{\Omega} \times \boldsymbol{R}^{n} \times \boldsymbol{R}^{n^{2}}$ to $\boldsymbol{R}^{n}$ and

$$
\int_{\Omega} \boldsymbol{g}(x, \boldsymbol{u}, \nabla \boldsymbol{u}) \cdot \boldsymbol{u} d x=0, \boldsymbol{u} \in \stackrel{\circ}{ }^{1}(\Omega), \nabla \cdot \boldsymbol{u}=0, n=2 .
$$

If $n=2, u \in \stackrel{\circ}{H}^{1}(\Omega)$ we have

(NS 2): $\quad \int_{\Omega}(\boldsymbol{g}(x, \boldsymbol{u}, \nabla \boldsymbol{u})-\boldsymbol{g}(x, \boldsymbol{v}, \nabla \boldsymbol{v})) \cdot(u-\boldsymbol{v}) d x \geqq-\varepsilon\|\boldsymbol{u}-\boldsymbol{v}\|_{1,2}^{2}$

$$
\begin{gathered}
-c(\varepsilon, C)\left(\|\boldsymbol{u}\|_{1,2}^{2}+\|\boldsymbol{v}\|_{1,2}^{2}\right)\|\boldsymbol{u}-\boldsymbol{v}\|_{L^{2}(\Omega)}^{2}, \\
\|\boldsymbol{u}\|_{L^{2}(\Omega)}+\|\boldsymbol{v}\|_{L^{2}(\Omega)} \leqq C, 1 \geqq \varepsilon>0 .
\end{gathered}
$$

(NS 3) $|g(x, \boldsymbol{u}, \nabla \boldsymbol{u})| \leqq c(n)\left(|\boldsymbol{u}|^{1+4 / n}+1+|\nabla \boldsymbol{u}|^{1+2 /(n+2)}\right), \quad n=2$,

where $\boldsymbol{u}, \boldsymbol{v} \in H^{1}(\Omega)$. (NS 3) follows from Hölder's inequality. As for (NS 2) we have by (I. 1)

$$
\begin{gathered}
\int_{\Omega}(\boldsymbol{u} \cdot \nabla \boldsymbol{u}-\boldsymbol{v} \cdot \nabla \boldsymbol{v}) \cdot(\boldsymbol{u}-\boldsymbol{v}) d x \geqq-\varepsilon \int_{\Omega}|\nabla(\boldsymbol{u}-\boldsymbol{v})|^{2} d x-c(\varepsilon)\left(\int _ { \Omega } \left(|\boldsymbol{u}|^{4}\right.\right. \\
\left.\left.+|\boldsymbol{v}|^{4}\right) d x\right)^{1 / 2}\left(\int_{\Omega}|\boldsymbol{u}-\boldsymbol{v}|^{4} d x\right)^{1 / 2} \geqq-\varepsilon\|\nabla(\boldsymbol{u}-\boldsymbol{v})\|_{L^{2}(\Omega)}^{2} \\
-c(\varepsilon, C)\left(\|\nabla \boldsymbol{u}\|_{L^{2}(\Omega)}^{2}+\|\nabla \boldsymbol{v}\|_{L^{2}(\Omega)}^{2}\right)\|\boldsymbol{u}-\boldsymbol{v}\|_{L^{2}(\Omega)}^{2} \cdot
\end{gathered}
$$

Our main result consists in

Theorem I.1. Let $n=2$, let $(\boldsymbol{u}, p)$ be a weak solution to the problem (NS). Then it is a strong solution.

Proof. Let us consiner the problems

$\left(\mathrm{NS}_{\sigma}\right)$

$$
\begin{aligned}
\boldsymbol{u}^{\prime}-\boldsymbol{\nu} \Delta \boldsymbol{u}+\boldsymbol{u} \cdot \nabla \boldsymbol{u}+\nabla p & =\sigma \boldsymbol{f}, \\
\nabla \cdot \boldsymbol{u} & =0, \\
\boldsymbol{u} \mid \partial \Omega & =0, \\
\boldsymbol{u}(0)=\sigma \boldsymbol{\phi}, \quad 0 \leqq \sigma & \leqq 1 .
\end{aligned}
$$

We introduce two sets $\Sigma, \Sigma^{*} \subset[0,1] . \Sigma$ is the set of all $\sigma$ for which $\left(\mathrm{NS}_{\sigma}\right)$ has a strong solution, $\Sigma^{*}$ is the set of all $\sigma$ with 
1. $[0, \sigma] \subset \Sigma$

2. $\int_{0}^{T}\left\|\boldsymbol{u}_{\tau}^{\prime}(t)\right\|_{L^{2}(\Omega)}^{2} d t+\underset{0 \leq t \leq T}{\operatorname{ess} \sup }\left\|\boldsymbol{u}_{\tau}(t)\right\|_{1,2}^{2}$

$$
\begin{aligned}
& +\int_{0}^{T}\left\|\boldsymbol{u}_{\tau}(t)\right\|_{2,2}^{2} d t+\int_{0}^{T}\left\|p_{\tau}(t)\right\|_{1,2}^{2} d t \\
\leqq & C(\sigma, \Omega, \nu)\left(\int_{0}^{T}\|\boldsymbol{f}(t)\|_{L^{2}(\Omega)}^{2} d t+1+\|\boldsymbol{\phi}\|_{1,2}^{2}\right), \quad 0 \leqq \tau \leqq \sigma .
\end{aligned}
$$

Obviously $0 \in \Sigma, \Sigma^{*}$.

Now let $\sigma_{1} \in \Sigma^{*}, \sigma_{2} \in \Sigma, \sigma_{2}>\sigma_{1}$. Using (NS 2) we get by scalar multiplication of our equations for $\boldsymbol{u}_{\sigma_{2}}-\boldsymbol{u}_{\sigma_{1}}$ with $\boldsymbol{u}_{\sigma_{2}}-\boldsymbol{u}_{\sigma_{1}}$ :

$$
\begin{gathered}
\left\|\boldsymbol{u}_{\sigma_{2}}(t)-\boldsymbol{u}_{\sigma_{1}}(t)\right\|_{L^{2}(\Omega)}^{2}+(\nu-\varepsilon) \int_{0}^{t}\left\|\nabla\left(\boldsymbol{u}_{\sigma_{2}}-\boldsymbol{u}_{\sigma_{1}}\right)(\tau)\right\|_{L^{2}(\Omega)}^{2} d t \\
\leqq \\
\int_{0}^{t}\left|\sigma_{2}-\sigma_{1}\right|\|\boldsymbol{f}(\tau)\|_{L^{2}(\Omega)}\left\|\boldsymbol{u}_{\sigma_{2}}(\tau)-\boldsymbol{u}_{\sigma_{1}}(\tau)\right\|_{L^{2}(\Omega)} d \tau \\
\quad+\int_{0}^{t} c(\varepsilon, C)\left(\left\|\nabla \boldsymbol{u}_{\sigma_{2}}(\tau)\right\|_{L^{2}(\Omega)}^{2}+\left\|\nabla \boldsymbol{u}_{\sigma_{1}}(\tau)\right\|_{L^{2}(\Omega)}^{2}\right) . \\
\left\|\boldsymbol{u}_{\sigma_{2}}(\tau)-\boldsymbol{u}_{\sigma_{1}}(\tau)\right\|_{L^{2}(\Omega)}^{2} d \tau+\left|\sigma_{2}-\sigma_{1}\right|\|\phi\|_{L^{2}(\Omega)}^{2}, \quad t \in[0, T] .
\end{gathered}
$$

The possibility of choosing a uniform $C$ is seen as follows: Using (NS 1) we get by scalar multiplication of our equation for $\boldsymbol{u}_{\sigma}$ with $\boldsymbol{u}_{\boldsymbol{\sigma}}$

$$
\begin{aligned}
& \left\|\boldsymbol{u}_{o}(t)\right\|_{L^{2}(\Omega)}^{2}+\nu \int_{0}^{t}\left\|\nabla \boldsymbol{u}_{\sigma}(\tau)\right\|_{L^{2}(\Omega)}^{2} d \tau \\
& \quad \leqq \int_{0}^{t}\|\boldsymbol{f}(\tau)\|_{L^{2}(\Omega)}\left\|\boldsymbol{u}_{\sigma}(\tau)\right\|_{L^{2}(\Omega)} d \tau+\|\boldsymbol{\phi}\|_{L^{2}(\Omega)}^{2}, \quad t \in[0,1], \sigma \in \Sigma .
\end{aligned}
$$

Thus we see that we can bound the $L^{1}((0, T))$-norm of $\left\|\nabla \boldsymbol{u}_{\sigma}(t)\right\|_{L^{2}(\Omega)}^{2}$ and the $L^{\infty}((0, T))$-norm of $\left\|\boldsymbol{u}_{\sigma}(t)\right\|_{L^{2}(\Omega)}$ independently of $\sigma$. Combining this with the previous inequality we see that

$$
\left\|\boldsymbol{u}_{\sigma_{1}}(t)-\boldsymbol{u}_{\sigma_{2}}(t)\right\|_{L^{2}(\Omega)} \leqq\left|\sigma_{1}-\sigma_{2}\right| c\left(\nu,\|\boldsymbol{\phi}\|_{L^{2}(\Omega)},\|\boldsymbol{f}\|_{L^{2}((0, T))}\right) .
$$

On using the lemma proved after Remark I.3 below we get

$$
\begin{aligned}
& \int_{0}^{T}\left\|\boldsymbol{u}_{\sigma_{1}}^{\prime}(t)-\boldsymbol{u}_{\sigma_{2}}^{\prime}(t)\right\|_{L^{2}(\Omega)}^{2} d t+\underset{0 \leqq t \leqq T}{\operatorname{ess} \sup }\left\|\boldsymbol{u}_{\sigma_{1}}(t)-\boldsymbol{u}_{\sigma_{2}}(t)\right\|_{1,2}^{2} \\
& \quad+\int_{0}^{T}\left\|\boldsymbol{u}_{\sigma_{1}}(t)-\boldsymbol{u}_{\sigma_{2}}(t)\right\|_{2,2}^{2} d t+\int_{0}^{T}\left\|p_{\sigma_{1}}(t)-p_{\sigma_{2}}(t)\right\|_{1,2}^{2} d t \\
& \leqq \\
& \quad c(\nu)\left(\left.\left|\sigma_{1}-\sigma_{2}\right|\right|_{0} ^{T}\|\boldsymbol{f}(t)\|_{L^{2}(\Omega)}^{2} d t+\left|\sigma_{1}-\sigma_{2}\right|\|\boldsymbol{\phi}\|_{1,2}^{2}\right. \\
& \left.\quad+\int_{0}^{T}\left\|\boldsymbol{u}_{\sigma_{1}} \cdot \nabla \boldsymbol{u}_{\sigma_{1}}(t)-\boldsymbol{u}_{\sigma_{2}} \cdot \nabla \boldsymbol{u}_{\sigma_{2}}(t)\right\|_{L^{2}(\Omega)}^{2} d t\right) .
\end{aligned}
$$

We have by Soboler ([1], p. 27) 


$$
\begin{aligned}
&\left\|\boldsymbol{u}_{\sigma_{1}} \cdot \nabla \boldsymbol{u}_{\sigma_{1}}(t)-\boldsymbol{u}_{\sigma_{2}} \cdot \nabla \boldsymbol{u}_{\sigma_{2}}(t)\right\|_{L^{2}(\Omega)} \\
& \leqq\left\|\boldsymbol{u}_{\sigma_{1}} \cdot \nabla \boldsymbol{u}_{\sigma_{1}}(t)\right\|_{L^{2}(\Omega)}+\left\|\boldsymbol{u}_{\sigma_{2}} \cdot \nabla \boldsymbol{u}_{\sigma_{2}}(t)\right\|_{L^{2}(\Omega)}, \\
& \leqq c(n)\left(\left\|\left.|| \boldsymbol{u}_{\sigma_{2}}\right|^{1+4 / n}\right\|_{L^{2}(\Omega)}+\left\|\left|\nabla \boldsymbol{u}_{\sigma_{2}}\right|^{1+2 /(n+2)}\right\|_{L^{2}(\Omega)}\right. \\
&+\left\|\boldsymbol{u}_{\sigma_{1}} \nabla \boldsymbol{u}_{\sigma_{1}}(t)\right\|_{L^{2}(\Omega)}, \\
& \leqq c(n)\left(\left\|\left|\boldsymbol{u}_{\sigma_{2}}-\boldsymbol{u}_{\sigma_{1}}\right|^{1+4 / n}\right\|_{L^{2}(\Omega)}+\left\|\left|\nabla \boldsymbol{u}_{\sigma_{2}}-\nabla \boldsymbol{u}_{\sigma_{1}}\right|^{1+2 /(n+2)}\right\|_{L^{2}(\Omega)}\right. \\
&\left.+\left\|\left.\boldsymbol{u}_{\sigma_{1}}\right|^{1+4 / n}\right\|_{L^{2}(\Omega)}+\left\|\left.\nabla \boldsymbol{u}_{\sigma_{1}}\right|^{1+2 /(n+2)}\right\|_{L^{2}(\Omega)}\right) \\
& \leqq c(n, \Omega)\left(\left\|\boldsymbol{u}_{\sigma_{2}}-\boldsymbol{u}_{\sigma_{1}}\right\|_{2,2}\left\|\boldsymbol{u}_{\sigma_{2}}-\boldsymbol{u}_{\sigma_{1}}\right\|_{L^{2}(\Omega)}^{4 / n}\right. \\
&+\left\|\boldsymbol{u}_{\sigma_{2}}-\boldsymbol{u}_{\sigma_{1}}\right\|_{2,2}\left\|\boldsymbol{u}_{\sigma_{2}}-\boldsymbol{u}_{\sigma_{1}}\right\|_{L^{2}(\Omega)}^{2 / n+2)} \\
&\left.+\left\|\left.\boldsymbol{u}_{\sigma_{1}}\right|^{1+4 / n}\right\|_{L^{2}(\Omega)}+\left\|\left|\nabla \boldsymbol{u}_{\sigma_{1}}\right|^{1+2 /(n+2)}\right\|_{L^{2}(\Omega)}\right) .
\end{aligned}
$$

Thus we see that in view of (I.3) $\boldsymbol{u}_{o_{2}}$ can be estimated as in part 2 of the definition of $\Sigma^{*}$ if $\left|\sigma_{1}-\sigma_{2}\right| \leqq \delta=\delta\left(n, \nu, \Omega,\|f\|_{L^{2}((O, T))}\right.$, $\left.\|\boldsymbol{\phi}\|_{L^{2}(\Omega)}\right)$. Observe that $\delta$ does not depend on $\sigma_{1}$. Now we want to prove that $\left[0, \sigma_{1}+\delta\right] \cap \Sigma^{*}=\left[0, \sigma_{1}+\delta\right]$. Let $\tau \in[0,1], \sigma \in\left[0, \sigma_{1}+\delta\right]$. Let $\boldsymbol{w}$ be an element of $W=\left\{\boldsymbol{w} \mid \boldsymbol{w}^{\prime} \in L^{2}\left((0, T), L^{2}(\Omega)\right), w \in L^{2}((0, T)\right.$, $\left.\left.H^{2}(\Omega)\right) \cap L^{\infty}\left((0, T), H^{1}(\Omega)\right)\right\}$, $\left(\tilde{T} w, p_{w}\right)$ the uniquely determined element of $V \times L^{2}\left((0, T), H^{1}(\Omega)\right)$ (cf. the lemma) with

$$
\begin{gathered}
(\widetilde{T} w)^{\prime}-\nu \Delta \widetilde{T} w+w \cdot \nabla w+\nabla p_{w}=\sigma f \\
\nabla \cdot \widetilde{T} w=0, \\
\widetilde{T} w(0)=\sigma \phi .
\end{gathered}
$$

Let $\left\{\boldsymbol{w}_{\nu}\right\}$ be a sequence from $K=\left\{\boldsymbol{w} \mid \boldsymbol{w} \in W\right.$, ess $\sup _{0 \leqq t \leqq T}\|\boldsymbol{w}(t)\|_{1,2}+$ $\left.\int_{0}^{T}\left\|\boldsymbol{w}^{\prime}(t)_{L^{2}(\Omega)}^{2} d t+\int_{0}^{T}\right\| \boldsymbol{w}(t) \|_{2,2}^{2} d t \leqq 1\right\}$ with

$$
\begin{aligned}
& \boldsymbol{w}_{\nu}^{\prime} \rightarrow \boldsymbol{w}^{\prime} \quad \text { in } \quad L^{2}\left((0, T), L^{2}(\Omega)\right), \\
& \boldsymbol{w}_{\nu} \rightarrow \boldsymbol{w} \quad \text { in } \quad L^{2}\left((0, T), H^{2}(\Omega)\right) .
\end{aligned}
$$

By Sobolev we have

$$
\begin{aligned}
& \int_{Q_{T}}\left|\boldsymbol{w}_{\nu}\right|^{2}\left|\nabla \boldsymbol{w}_{\nu}-\nabla \boldsymbol{w}_{\mu}\right|^{2} d x d t \leqq\left(\int_{0}^{T}\left\|\boldsymbol{w}_{\nu}\right\|_{L^{2 q_{1}(\Omega)}}^{2 q_{1}} d t\right)^{1 / q_{1}} \\
& \cdot\left(\int_{0}^{T}\left\|\nabla \boldsymbol{w}_{\nu}-\nabla \boldsymbol{w}_{\mu}\right\|_{L^{2 q_{2}(\Omega)}}^{2 q_{2}} d t\right)^{1 / q_{2}} \\
& \leqq\left(\int_{0}^{T}\left\|\boldsymbol{w}_{\nu}\right\|_{L^{2 q_{1}(\Omega)}}^{2 q_{1}} d t\right)^{1 / q_{1}}\left(\int_{0}^{T}\left\|\boldsymbol{w}_{\nu}-\boldsymbol{w}_{\mu}\right\|_{2,2}^{\alpha 2 p_{2}}\left\|\boldsymbol{w}_{\nu}-\boldsymbol{w}_{\mu}\right\|_{L^{q}(\Omega)}^{(1-\alpha) 2 q_{2}} d t\right)^{1 / q_{2}} \\
& \leqq\left(\int_{0}^{T}\left\|\boldsymbol{w}_{\nu}\right\|_{L^{2 q_{1}(\Omega)}}^{2 q_{1}} d t\right)^{1 / q_{1}}\left(\int_{0}^{T}\left\|\boldsymbol{w}_{\nu}-\boldsymbol{w}_{\mu}\right\|_{2,2}^{\alpha 2 p_{2} p_{1}} d t\right)^{1 / q_{2} p_{1}} \\
& \cdot\left(\int_{0}^{T}\left\|\boldsymbol{w}_{\nu}-\boldsymbol{w}_{\mu}\right\|_{L^{q}(\Omega)}^{(1-\alpha) 2 q_{2} p_{1}} d t\right)^{1 / q_{2} p_{1}}
\end{aligned}
$$

Here 


$$
\frac{1}{q_{1}}+\frac{1}{q_{2}}=1=\frac{1}{p_{1}}+\frac{1}{p_{2}}, \frac{1}{2 q_{2}}=\frac{1}{2}+(1-\alpha) \frac{1}{q}-\frac{\alpha}{2} .
$$

It is easily verified that we can choose $q_{2}, p_{1}, \alpha, p_{2}, q$ in such a way that the relations above and the following ones are fulfilled: $1<q<6, \quad(1-\alpha) 2 q_{2} p_{1}<6, \alpha 2 q_{2} p_{1} \leqq 2$. Now we have to observe that $H^{1}(\Omega) \subset L^{p}(\Omega), \mathrm{p} \geqq 1$, with a continuous imbedding and that $H^{1}\left(Q_{T}\right) \subset L^{q}\left(Q_{T}\right), q<6$, with a completely continuous imbedding. Since the term $\int_{Q_{T}}\left|\boldsymbol{w}_{\mu}-\boldsymbol{w}_{\nu}\right|^{2}\left|\nabla \boldsymbol{w}_{\mu}\right|^{2} d x d t$ can be treated quite similarly we see that $\int_{0}^{T}\left\|\boldsymbol{w}_{\nu} \nabla \boldsymbol{w}_{\nu}-\boldsymbol{w}_{\mu} \nabla \boldsymbol{w}_{\mu}\right\|_{L^{2}(\Omega)}^{2} d t \rightarrow 0, \nu, \mu \rightarrow \infty$. So far we have shown that $\widetilde{T}$ is a completely continuous mapping of $W$ in itself. Moreover, we have just proved that all fixed points of $\tau \widetilde{T}, 0 \leqq \tau \leqq$ 1 , remain uniformly bounded in the norm of $W$. Schaefer's fixed point theorem now yields that $\left[0, \sigma_{1}+\delta\right] \cap \Sigma^{*}=\left[0, \sigma_{1}+\delta\right]$. Since $\delta$ does not depend on $\sigma_{1}$ we finally arrive at $[0,1]=\Sigma=\Sigma^{*}$. Thus our theorem is proved.

REMARK I.1. The proof of Theorem I. 1 of course yields an $a$ priori estimate for every strong solution (cf. the introduction of $\Sigma^{*}$ ). Probably $\Omega$ can be unbounded.

REMARK I. 2. Using the same method as in the preceding theorem one can construct strong solutions to the Navier-Stokes equations with the property

$$
\begin{aligned}
& \boldsymbol{u}^{\prime} \in L^{p}\left((0, T), L^{p}(\Omega)\right), \\
& \boldsymbol{u} \in L^{p}\left((0, T), H^{2, p}(\Omega)\right) \cap L^{p}\left((0, T), \stackrel{\circ}{H}^{1, p}(\Omega)\right), \\
& p \in L^{p}\left((0, T), H^{1, p}(\Omega)\right)
\end{aligned}
$$

if $f \in L^{p}\left((0, T), \quad L^{p}(\Omega)\right), \quad \phi \in H^{2, p}(\Omega) \cap \dot{H}^{1, p}(\Omega), \quad p \geqq 2$. The reason is simply that

$$
\begin{gathered}
\left\||\boldsymbol{u}|^{1+4 / n}\right\|_{L^{p}(\Omega)} \leqq c(n, p, \Omega)\|\boldsymbol{u}\|_{2, p}\|\boldsymbol{u}\|_{L^{2}(\Omega)}^{4 / n}, \\
\left\||\nabla \boldsymbol{u}|^{1+2 /(n+2)}\right\|_{L^{p}(\Omega)} \leqq c(n, p, \Omega)\|\boldsymbol{u}\|_{2, p}\|\boldsymbol{u}\|_{L^{2}(2)}^{2 / 2(2)}, \quad \boldsymbol{u} \in H^{2, p}(\Omega),
\end{gathered}
$$

and that we have estimates in $L^{p}$-spaces corresponding to that of our lemma (see [13], Ch. IV). An analogous result for semilinear parabolic systems will be proved by a very similar method in the next chapter. We want to state our a priori estimate for strong solutions of Navier-Stokes equations in the following theorem:

THEOREM I.2. Let $T>0$ be arbitrary. Let $f \in L^{p}\left((0, T), L^{p}(\Omega)\right)$, $p \geqq 2$, let $\boldsymbol{\phi} \in H^{2, p}(\Omega) \cap \stackrel{\circ}{H}^{1, p}(\Omega)$. Let $\boldsymbol{u} \in L^{p}\left((0, T), H^{2, p}(\Omega)\right) \cap L^{p}((0, T)$, $\left.\dot{H}^{1, p}(\Omega)\right), \quad \boldsymbol{u}^{\prime} \in L^{p}\left((0, T), \quad L^{p}(\Omega)\right), \quad p \in L^{p}\left((0, T), H^{1, p}(\Omega)\right)$ and let $(\boldsymbol{u}, p)$ 
fulfill the equations

$$
\begin{aligned}
\boldsymbol{u}^{\prime}-\boldsymbol{\nu} \Delta \boldsymbol{u}+\boldsymbol{u} \cdot \nabla \boldsymbol{u}+\nabla p & =\boldsymbol{f}, \\
\nabla \cdot \boldsymbol{u} & =0, \\
\boldsymbol{u}(0) & =\boldsymbol{\phi} .
\end{aligned}
$$

Then the following a priori estimate holds:

$$
\begin{aligned}
& \int_{0}^{T}\left\|\boldsymbol{u}^{\prime}(t)\right\|_{L^{p}(\Omega)}^{p} d t+\int_{0}^{T}\|\boldsymbol{u}(t)\|_{2, p}^{p} d t+\int_{0}^{T}\|\nabla p(t)\|_{L^{p}(\Omega)}^{p} d t \\
& \quad+\underset{0 \leqq t \leqq T}{\operatorname{ess} \sup }\|\boldsymbol{u}(t)\|_{1,2} \leqq c(\nu 1, T)\left(\|\boldsymbol{\phi}\|_{2, p}^{p}+\int_{0}^{T}\|\boldsymbol{f}(t)\|_{L^{p}(\Omega)}^{p} d t\right) .
\end{aligned}
$$

For $p=2$ it is sufficient to assume that $\phi \in \stackrel{\circ}{H}^{1}(\Omega)$ and $\left\|\boldsymbol{\phi}_{2, p}\right\|$ in the estimate above may be replaced by $\|\boldsymbol{\phi}\|_{1,2}$.

REMARK I.3. Instead of boundary values 0 one can prescribe boundary values $\boldsymbol{a}$ with $\boldsymbol{a} \in W, \nabla \cdot \boldsymbol{a}=0, \int_{\partial \Omega} \boldsymbol{a} \cdot \boldsymbol{n} d \omega=0$, where $\boldsymbol{n}$ is the outer normal on $\partial \Omega$.

We now prove the lemma mentioned in the proof of Theorem I.1.

LEMma. Let $f \in L^{2}\left((0, T), L^{2}(\Omega)\right), \phi \in \stackrel{H}{H}^{1}(\Omega)$. Then there exists a pair $(\boldsymbol{u}, p)$ such that $\boldsymbol{u} \in L^{2}\left((0, T), H^{2}(\Omega)\right) \cap L^{\infty}\left((0, T), \dot{H}^{1}(\Omega)\right), \boldsymbol{u}^{\prime} \epsilon$ $L^{2}\left((0, T), L^{2}(\Omega)\right), p \in L^{2}\left((0, T), H^{1}(\Omega)\right)$,

$$
\begin{aligned}
\boldsymbol{u}^{\prime}-\nu \Delta \boldsymbol{u}+\nabla p & =\boldsymbol{f}, \\
\nabla \boldsymbol{u} & =\mathbf{0}, \\
\boldsymbol{u}(0) & =\boldsymbol{\phi},
\end{aligned}
$$

and the following a-priori estimate holds:

$$
\begin{aligned}
& \int_{0}^{T}\left\|\boldsymbol{u}^{\prime}(t)\right\|_{L^{2}(\Omega)}^{2} d t+\underset{0 \leqq t \leqq T}{\operatorname{ess} \sup }\|\boldsymbol{u}(t)\|_{1,2}^{2}+\int_{0}^{T}\|\boldsymbol{u}(t)\|_{2,2}^{2} d t \\
& \quad+\int_{0}^{T}\|p(t)\|_{1,2}^{2} d t \leqq c(\nu, n, \Omega)\left(\|\boldsymbol{\phi}\|_{1,2}^{2}+\int_{0}^{T}\|\boldsymbol{f}(t)\|_{L^{2}(\Omega)}^{2} d t\right) .
\end{aligned}
$$

Proof. Approximating $p$ we see that

$$
\int_{0}^{T}\left(\nabla p, \boldsymbol{u}^{\prime}\right) d t=0 \text {. }
$$

Thus scalar multiplication of (I.4) with $\boldsymbol{u}^{\prime}$ yields the desired $a$ priori estimate for

$$
\int_{0}^{T}\left\|\boldsymbol{u}^{\prime}(t)\right\|_{L^{2}(\Omega)}^{2} d t+\underset{0 \leqq t \leqq T}{\operatorname{ess} \sup }\|\boldsymbol{u}(t)\|_{1,2}^{2} .
$$


Let us consider the Stokes-system

$$
-\boldsymbol{\nu} \Delta \boldsymbol{u}+\nabla p=-\boldsymbol{u}^{\prime}+\boldsymbol{f} .
$$

Application of the results of $\S 4, \S 5$, Ch. III in [3] finally yields the desired estimate and therefore the lemma.

REMARK I.4. It is evident, the lemma above implies that for every $\boldsymbol{f} \in L^{2}\left((0, T), L^{2}(\Omega)\right)$ and every $\phi \in \dot{H}^{1}(\Omega)$ with $\nabla \cdot \phi=0$ there exists a unique pair $(\boldsymbol{u}, p)$ with $\boldsymbol{u} \in L^{2}\left((0, T), \quad H^{2}(\Omega)\right) \cap L^{\infty}((0, T)$, $\dot{H}^{1}((\Omega)), \boldsymbol{u}^{\prime} \in L^{2}\left((0, T), L^{2}(\Omega)\right), p \in L^{2}\left((0, T), H^{1}(\Omega)\right)$ and (I.4), (I.4').

II. Parabolic System. In this section we want to carry over our results from $\S I$ to parabolic systems in diagonal form. Let $T>0$ but otherwise arbitrary, let $\Omega$ be a bounded open set of $\boldsymbol{R}^{n}$ whose boundary is of class $C^{4 m}$ for an $m \in N$. For each pair of multi-indices $\tilde{\alpha}, \widetilde{\beta}$ of $\boldsymbol{R}^{n}$ with $|\widetilde{\alpha}|,|\widetilde{\beta}| \leqq m$, let functions

$$
A_{\tilde{\alpha} \tilde{\beta}}:[0, T] \times \bar{\Omega} \longrightarrow C
$$

be given with

$$
\begin{aligned}
& A_{\tilde{\alpha} \tilde{\beta}} \in C^{0}\left([0, T], \quad C^{m}(\bar{\Omega})\right), \\
& (1 / i)^{\mid \widetilde{|\alpha|+|\widetilde{\beta}|}} A_{\tilde{\alpha} \tilde{\beta}}(t, x) \in R .
\end{aligned}
$$

Moreover we assume that

$$
M|\xi|^{2 m} \geqq \sum_{\substack{|\tilde{\alpha}=m,\\| \tilde{\beta} \mid=m}} A_{\tilde{\alpha} \tilde{\beta}}(t, x) \xi^{\tilde{\alpha}+\tilde{\beta}} \geqq M^{-1}|\xi|^{2 m}, \quad(t, x) \in[0, T] \times \bar{\Omega}, \xi \in \boldsymbol{R}^{n},
$$

with a positive constant $M$. We set for $p>1$

$$
A_{p}(t) u=\sum_{\substack{|\tilde{\alpha}| \leq m,|\tilde{\beta}| \leq m}} D^{\tilde{\alpha}}\left(A_{\tilde{\alpha} \tilde{\beta}}(t, x) D^{\tilde{\beta}} u\right), \quad u \in H^{2 m, p}(\Omega) \cap \dot{H}^{m, p}(\Omega) .
$$

For our purposes it means no loss of generality if we assume that Garding's inequality

$$
\left(A_{2}(t) u, u\right) \geqq c\left(\Omega, m, n, M,\left\||| A_{\tilde{\alpha} \tilde{\beta}}\left|\|, \quad\|\left\|\left|A_{\tilde{\alpha} \tilde{\beta}}\right|\right\| \|_{x}\right)\right\| u \|_{m, 2}^{2}\right.
$$

holds with a positive constant $c(\Omega, m, n, \cdots)$.

Let $\boldsymbol{f}$ be a mapping from $[0, T] \times \bar{\Omega} \times \boldsymbol{R}^{l} \times \boldsymbol{R}^{l n_{l}} \times \cdots \times \boldsymbol{R}^{l n_{m}}$ $\left(n_{\nu}=\right.$ number of multi-indices $\tilde{\alpha}$ with $\left.|\tilde{\alpha}|=\nu\right)$ in $\boldsymbol{R}^{1}$, where $l$ is a fixed number. $\boldsymbol{f}$ is supposed to have the following properties:

$\boldsymbol{f}$ is Lipschitz continuous and $\boldsymbol{f}(t, x, 0, \cdots, 0)=0$,

$$
\int_{\Omega} \boldsymbol{f}\left(t, x, \boldsymbol{u}, \nabla \boldsymbol{u}, \cdots, \nabla^{m} \boldsymbol{u}\right) \cdot \boldsymbol{u} d x \geqq 0, \quad \boldsymbol{u} \in \stackrel{\circ}{H}^{m}(\Omega) .
$$


In particular $\hat{\boldsymbol{u}}, \boldsymbol{f}\left(t, \nabla \boldsymbol{u}, \cdots, \nabla^{m} \boldsymbol{u}\right)$ is supposed to be integrable for $\boldsymbol{u} \in H^{m}(\Omega), \hat{\boldsymbol{u}} \in H^{m}(\Omega)$.

$$
\begin{gathered}
\int_{\Omega}\left(\boldsymbol{f}\left(t, x, \boldsymbol{u}, \nabla \boldsymbol{u}, \cdots, \nabla^{m} \boldsymbol{u}\right)-\boldsymbol{f}\left(t, x, \boldsymbol{u}, \nabla \boldsymbol{v}, \cdots, \nabla^{m} \boldsymbol{v}\right)\right) \cdot(\boldsymbol{u}-\boldsymbol{v}) d x \\
\quad \geqq-\varepsilon\|\boldsymbol{u}-\boldsymbol{v}\|_{m, 2}^{2}-c(\varepsilon, C)\left(\|\boldsymbol{u}\|_{m, 2}^{2}+\|\boldsymbol{v}\|_{m, \mathbf{2}}^{2}\right)\|\boldsymbol{u}-\boldsymbol{v}\|_{L^{2}(\Omega)}^{2}
\end{gathered}
$$

for all $\varepsilon, 1 \geqq \varepsilon>0, C \geqq\|\boldsymbol{u}\|_{L^{2}(\Omega)}+\|\boldsymbol{v}\|_{L^{2}(\Omega)}$.

$$
\begin{gathered}
\left|\frac{\partial^{\lambda}}{\partial p_{\nu \mu}^{\lambda}} \boldsymbol{f}\left(t, x, \boldsymbol{p}_{0}, \cdots, \boldsymbol{p}_{n_{m}}\right)\right| \leqq K\left(1+\sum_{\nu=0}^{m}\left|\boldsymbol{p}_{\nu}\right|^{1+(4 m-2 \nu) /(n+2 \nu)-\lambda}\right), \\
\lambda=0,1,(t, x) \in[0, T] \times \bar{\Omega}, \boldsymbol{p}_{\nu} \in \boldsymbol{R}^{1 n_{\nu}}-\{0\}, \\
\boldsymbol{p}_{\nu}=\left(p_{\nu_{1}}, \cdots, \boldsymbol{p}_{\nu_{l n_{\nu}}}\right) .
\end{gathered}
$$

As an example we can take the nonlinearity with components

$$
f^{\lambda}\left(t, x, \boldsymbol{u}, \nabla \boldsymbol{u}, \cdots, \nabla^{m} \boldsymbol{u}\right)=u^{\lambda}\left|\nabla^{m} \boldsymbol{u}\right|, \quad 1 \leqq \lambda \leqq l,
$$

if we assume that $n \leqq 2 m$. If one uses the Sobolev-inequality

$$
\|\boldsymbol{u}\|_{L^{4}(\Omega)} \leqq c(n, m, \Omega)\|\boldsymbol{u}\|_{m, 2}^{\alpha}\|\boldsymbol{u}\|_{L^{2}(\Omega)}^{1-\alpha}
$$

with $\alpha=n / 4 m \leqq 1 / 2$ the validity of (F3) for the nonlinearity (II.1) can be shown in the same way as we did with (NS 2). As for (F 4) we have

$$
\begin{aligned}
& \left|u^{2}\right| \nabla^{m} \boldsymbol{u}|| \leqq|\boldsymbol{u}|\left|\nabla^{m} \boldsymbol{u}\right| \\
& \quad \leqq c(n, m)\left(|\boldsymbol{u}|^{1+4 m / n}+\left|\nabla^{m} \boldsymbol{u}\right|^{1+n / 4 m}\right), \\
& \quad \leqq c(n, m)\left(|\boldsymbol{u}|^{1+4 m / n}+\left|\nabla^{m} \boldsymbol{u}\right|^{1+2 m /(n+2 m)}+1\right),
\end{aligned}
$$

since $1+n / 4 m \leqq 1+2 m /(n+2 m)$ if $n \leqq 4 m$.

Now we want to prove the main result of this section, namely.

THEOREM II. 1. Let $\boldsymbol{g} \in L^{p}\left((0, T), L^{p}(\Omega)\right)$ for $a \quad p \geqq 2$. Let $\phi \in H^{2 m, p}(\Omega) \cap \dot{H}^{m, p}(\Omega)$. Then there exists a unique u with the following properties:

$$
\begin{aligned}
& \boldsymbol{u}^{\prime} \in L^{p}\left((0, T), L^{p}(\Omega)\right), \\
& \boldsymbol{u} \in L^{p}\left((0, T), H^{2 m, p}(\Omega)\right) \cap L^{p}\left((0, T), \dot{H}^{m, p}(\Omega)\right), \\
& \boldsymbol{u}(0)=\Phi, \\
& \boldsymbol{u}^{\prime}+A(t) \boldsymbol{u}+\boldsymbol{f}\left(t, \boldsymbol{u}, \nabla \boldsymbol{u}, \cdots, \nabla^{m} \boldsymbol{u}\right)=\boldsymbol{g} .
\end{aligned}
$$

Proof. Since the proof is very similar to that of Theorem I.1 we only sketch it. $\Sigma, \Sigma^{*}$ can be introduced as in Theorem I.1. The decisive point is of course the estimate of $\boldsymbol{u}_{\sigma_{2}}-\boldsymbol{u}_{\sigma_{1}}, \sigma_{2}>\sigma_{1} \in$ $\Sigma^{*}$. Application of Theorem 10.4, Ch. VII in [4] yields: 


$$
\begin{aligned}
& \int_{0}^{T}\left\|\boldsymbol{u}_{\sigma_{2}}^{\prime}(t)-\boldsymbol{u}_{\sigma_{1}}^{\prime}(t)\right\|_{L^{p}(\Omega)}^{p} d t+\int_{0}^{T}\left\|\boldsymbol{u}_{\sigma_{2}}(t)-\boldsymbol{u}_{\sigma_{1}}(t)\right\|_{2 m, p}^{p} d t \\
& \leqq c\left(n, m, \Omega, p, M, T,\left\|A_{\alpha \tilde{\beta}}\right\|\|, \cdots,\| \nabla^{m} A_{\alpha \tilde{\beta}}\|\|, \quad\|\| A_{\alpha \tilde{\beta}}\|\| \|\right) \\
& \times\left(\int_{0}^{T}\left\|\boldsymbol{f}\left(t, \boldsymbol{u}_{\sigma_{1}}, \nabla \boldsymbol{u}_{\sigma_{1}}, \cdots, \nabla^{m} \boldsymbol{u}_{\sigma_{1}}\right)\right\|_{L^{p}(\Omega)}^{p} d t\right. \\
&+\int_{0}^{T}\left\|\boldsymbol{f}\left(t, \boldsymbol{u}_{\sigma_{2}}, \nabla \boldsymbol{u}_{\sigma_{2}}, \cdots, \nabla^{m} \boldsymbol{u}_{\sigma_{2}}\right)\right\|_{L^{p}(\Omega)}^{p} d t+\left|\sigma_{2}-\sigma_{1}\right|^{p} \int_{0}^{T}\|\boldsymbol{g}(t)\|_{L^{p}(\Omega)}^{p} d t \\
&\left.+\left|\sigma_{2}-\sigma_{1}\right|^{p}\|\phi\|_{2 m, p}\right) .
\end{aligned}
$$

As in Theorem I.1 we can prove that

$$
\begin{array}{r}
\left\|\boldsymbol{u}_{\sigma_{2}}(t)-\boldsymbol{u}_{\sigma_{1}}(t)\right\|_{L^{2}(\Omega)} \leqq\left|\sigma_{2}-\sigma_{1}\right| c\left(\int_{0}^{T}\|\boldsymbol{f}(t)\|_{L^{2}(\Omega)}^{2} d t,\right. \\
\left.\|\boldsymbol{\phi}\|_{L^{2}(\Omega)}, \Omega, n, m, M,\left\|A_{\tilde{\alpha} \tilde{\beta}}\right\|, \quad\|\| A_{\alpha \tilde{\beta}}^{\tilde{\alpha}}\|\|_{x}\right) .
\end{array}
$$

\section{Moreover}

$$
\begin{aligned}
& \int_{0}^{T}\left\|\boldsymbol{f}\left(t, \boldsymbol{u}_{\sigma_{2}}, \nabla \boldsymbol{u}_{\sigma_{2}}, \cdots, \nabla^{m} \boldsymbol{u}_{\sigma_{2}}\right)\right\|_{L^{p}(\Omega)}^{p} d t \\
& \leqq c(K, p, n, \Omega)\left(1+\sum_{\nu=0}^{m} \int_{0}^{T}\left\|\left|\nabla^{\nu}\left(\boldsymbol{u}_{\sigma_{2}}(t)-\boldsymbol{u}_{\sigma_{1}}(t)\right)\right|^{1+(4 m-2 \nu) /(n+2 \nu)}\right\|_{L^{p_{(}(\Omega)}}^{p} d t\right. \\
&\left.+\sum_{\nu=0}^{m} \int_{0}^{T}\left\|\left|\nabla^{\nu} \boldsymbol{u}_{\sigma_{1}}(t)\right|^{1+(4 m-2 \nu) /(n+2 \nu)}\right\|_{L^{p}(\Omega)}^{p} d t\right) .
\end{aligned}
$$

Since (cf. [1], p. 27)

$$
\begin{aligned}
& \left\|\left|\nabla^{\nu}\left(\boldsymbol{u}_{\sigma_{2}}(t)-\boldsymbol{u}_{\sigma_{1}}(t)\right)\right|^{1+(4 m-2 \nu) /(n+2 \nu)}\right\|_{L^{p}(\Omega)}^{p} \\
& \quad \leqq c(n, m, \Omega, p)\left\|\boldsymbol{u}_{\sigma_{2}}(t)-\boldsymbol{u}_{\sigma_{1}}(t)\right\|_{2 m, p}^{p}\left\|\boldsymbol{u}_{\sigma_{2}}(t) \boldsymbol{u}_{\sigma_{1}}(t)\right\|_{L^{2}(\Omega)}^{p(m-2 \nu) /(n+2 \nu)}
\end{aligned}
$$

we see that for $\left|\sigma_{2}-\sigma_{1}\right|$ sufficiently small (the bound does not depend on $\sigma_{1}$ ) the norm

$$
\int_{0}^{T}\left\|\boldsymbol{u}_{\sigma_{2}}^{\prime}(t)-\boldsymbol{u}_{\sigma_{1}}^{\prime}(t)\right\|_{L^{p}(\Omega)}^{p} d t+\int_{0}^{T}\left\|\boldsymbol{u}_{\sigma_{2}}(t)-\boldsymbol{u}_{\sigma_{1}}(t)\right\|_{2 m, p}^{p} d t
$$

can be estimated a priori. Thus the rest of the proof can be completed as the corresponding one in Chapter I.

\section{REFERENCES}

1. A. Friedman, Partial Differential Equations, Holt, Rinehart and Winston, NewYork-Chicago-San-Francisco, 1969.

2. H. Fujita and T. Kato, On the Navier-Stokes initial value problem I, Arch. Rational Mech. Anal., 16 (1964), 269-315.

3. O. A. Ladyženskaja, Funktionalanalytische Untersuchungen Über die Navier-Stokesschen Gleichungen, Akademie-Verlag, Berlin, 1964.

4. 0. A. Ladyženskaja, V. A. Solonnikov and N. N. Ural'ceva, Linear and Quasilinear Equations of Parabolic Type. Translations of Mathematical Monographs, 23, Providence; Amer. Math. Soc. 1968. 
5. J. L. Lions, Quelques Methodes de Résolution des Problèmes Nonlinéaires, Dunod, Paris, 1969.

6. M. Shinbrot, and S. Kaniel, Smoothness of weak solutions of the Navier-Stokes equations, Arch. Rational Mech. and Anal., 24, (1967), 302-324.

7. - The initial value problem for the Navier-Stokes equations, Arch. Rational Mech. Anal., 21, (1966), 270-285.

8. J. Serrin, The Initial Value Problem for the Navier-Stokes Equations, in Nonlinear Problems, Rudolph E. Langer, ed., University of Wisconsin Press, Madison, 1963.

Received December, 24, 1974.

MATHEMATISChES INSTITUT DER

RUHR-UNIVERSITÄT BOCHUM

UNIVERSITÄTSSTR, 150

D-4630 BоCHUM 1 



\title{
PACIFIC JOURNAL OF MATHEMATICS
}

\section{EDITORS}

\author{
RICHARD ARENS (Managing Editor) \\ University of California \\ Los Angeles, CA 90024 \\ Charles W. Curtis \\ University of Oregon \\ Eugene, OR 97403 \\ C. C. MOORE \\ University of California \\ Berkeley, CA 94720
}

\section{J. DUGUNDJI}

Department of Mathematics

University of Southern California

Los Angeles, CA 90007

R. FINN and J. MILGRAM

Stanford University

Stanford, CA 94305

\section{ASSOCIATE EDITORS}
E. F. BECKENBACH
B. H. NeumanN
F. WOLF
K. YoSHIDA

\section{SUPPORTING INSTITUTIONS}

UNIVERSITY OF BRITISH COLUMBIA

CALIFORNIA INSTITUTE OF TECHNOLOGY

UNIVERSITY OF CALIFORNIA

MONTANA STATE UNIVERSITY

UNIVERSITY OF NEVADA, RENO

NEW MEXICO STATE UNIVERSITY

OREGON STATE UNIVERSITY

UNIVERSITY OF OREGON

OSAKA UNIVERSITY

\author{
UNIVERSITY OF SOUTHERN CALIFORNIA \\ STANFORD UNIVERSITY \\ UNIVERSITY OF HAWAII \\ UNIVERSITY OF TOKYO \\ UNIVERSITY OF UTAH \\ WASHINGTON STATE UNIVERSITY \\ UNIVERSITY OF WASHINGTON \\ $\stackrel{*}{*} \stackrel{*}{*} \stackrel{*}{*}$ AMERICAN MATHEMATICAL SOCIETY
}

The Supporting Institutions listed above contribute to the cost of publication of this Journal, but they are not owners or publishers and have no responsibility for its content or policies.

Mathematical papers intended for publication in the Pacific Jaurnal of Mathematics should be in typed form or offset-reproduced, (not dittoed), double spaced with large margins. Please do not use built up fractions in the text of your manuscript. You may however, use them in the displayed equations. Underline Greek letters in red, German in green, and script in blue. The first paragraph or two must be capable of being used separately as a synopsis of the entire paper. Items of the bibliography should not be cited there unless absolutely necessary, in which case they must be identified by author and Journal, rather than by item number. Manuscripts, in triplicate, may be sent to any one of the editors. Please classify according to the scheme of Math. Reviews, Index to Vol. 39. All other communications should be addressed to the managing editor, or Elaine Barth, University of California, Los Angeles, California, 90024.

The Pacific Journal of Mathematics expects the author's institution to pay page charges, and reserves the right to delay publication for nonpayment of charges in case of financial emergency.

100 reprints are provided free for each article, only if page charges have been substantially paid. Additional copies may be obtained at cost in multiples of 50 .

The Pacific Journal of Mathematics is issued monthly as of January 1966. Regular subscription rate: $\$ 7200$ a year (6 Vols., 12 issues). Special rate: $\$ 36.00$ a year to individual members of supporting institutions.

Subscriptions, orders for back numbers, and changes of address should be sent to Pacific Journal of Mathematics, 103 Highland Boulevard, Berkeley, California, 94708.

PUBLISHED BY PACIFIC JOURNAL OF MATHEMATICS, A NON-PROFIT CORPORATION

Printed at Kokusai Bunken Insatsusha (International Academic Printing Co., Ltd.). 8-8, 3-chome, Takadanobaba, Shinjuku-ku, Tokyo 160, Japan.

Copyright (C) 1975 by Pacific Journal of Mathematics Manufactured and first issued in Japan 


\section{Pacific Journal of Mathematics}

Vol. 72, No. 2

February, 1977

George E. Andrews, Plane partitions. II. The equivalence of the

Bender-Knuth and MacMahon conjectures ................. 283

Lee Wilson Badger, An Ehrenfeucht game for the multivariable quantifiers

of Malitz and some applications ......................... 293

Wayne C. Bell, A decomposition of additive set functions ............ 305

Bruce Blackadar, Infinite tensor products of $C^{*}$-algebras ............. 313

Arne Brøndsted, The inner aperture of a convex set .............. 335

N. Burgoyne, Finite groups with Chevalley-type components........... 341

Richard Dowell Byrd, Justin Thomas Lloyd and Roberto A. Mena, On the retractability of some one-relator groups .....................

Paul Robert Chernoff, Schrödinger and Dirac operators with singular potentials and hyperbolic equations .................... 361

John J. F. Fournier, Sharpness in Young's inequality for convolution ....... 383

Stanley Phillip Franklin and Barbara V. Smith Thomas, On the metrizability

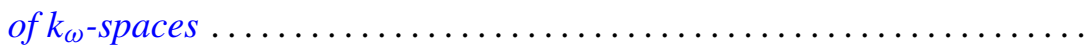

David Andrew Gay, Andrew McDaniel and William Yslas Vélez, Partially normal radical extensions of the rationals .................... 403

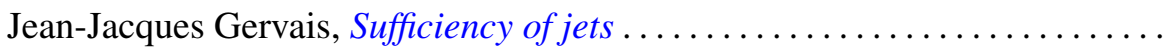

Kenneth R. Goodearl, Completions of regular rings. II . .

Sarah J. Gottlieb, Algebraic automorphisms of algebraic groups with stable maximal tori

Donald Gordon James, Invariant submodules of unimodular Hermitian forms.....

J. Kyle, $W_{\delta}(T)$ is convex.

Ernest A. Michael and Mary Ellen Rudin, A note on Eberlein compacts ...

Ernest A. Michael and Mary Ellen Rudin, Another note on Eberlein compacts ....

Thomas Bourque Muenzenberger and Raymond Earl Smithson, Fixed point theorems for acyclic and dendritic spaces.

Budh Singh Nashier and A. R. Rajwade, Determination of a unique solution of the quadratic partition for primes $p \equiv 1(\bmod 7)$.

Frederick J. Scott, New partial asymptotic stability results for nonlinear ordinary differential equations ....................

Frank Servedio, Affine open orbits, reductive isotropy groups, and dominant gradient morphisms; a theorem of Mikio Sato..........

D. Suryanarayana, On the distribution of some generalized square-full integers.................................. 